\title{
Stabilization of a Coupled Second Order ODE-wave System
}

\author{
ZHEN Zhiyuan ${ }^{1}$, WANG Ke ${ }^{2}$, ZHOU Zhongcheng ${ }^{1 *}$, Ryan LOXTON ${ }^{3}$ \\ 1. School of Mathematics and Statistics, Southwest University, Chongqing, China \\ E-mail: zhenzhiyuan@swu.edu.cn, zhouzc@amss.ac.cn \\ 2. Department of Information and Computational Science, Chengdu Technological University, Chengdu, China \\ E-mail: wke1@cdtu.edu.cn \\ 3. Department of Mathematics and Statistics, Curtin University, Perth, Australia \\ E-mail: r.loxton@curtin.edu.au
}

\begin{abstract}
This paper considers the stabilization of a coupled second order ODE-wave system, where the ODE dynamics contain the solution of the wave equation at an intermediate point. We design a stabilizing feedback controller by choosing a suitable target system and backstepping transformation. The backstepping transformation is defined in terms of several kernel functions, for which we establish existence, uniqueness and smoothness properties. We also prove exponential stability for the resulting closed-loop system. Finally, the effectiveness of the proposed feedback controller is verified via a numerical example.
\end{abstract}

Key Words: ODE-wave system, Backstepping, Stabilization, Control design

\section{Introduction}

In this paper, we consider the exponential stabilization of the following coupled second order ODE-wave system:

$$
\left\{\begin{array}{l}
y^{\prime \prime}(t)=a^{2} y(t)+b u\left(x_{0}, t\right), t>0, \\
u_{t t}(x, t)=u_{x x}(x, t), 0<x<1, t>0, \\
u(0, t)=0, u_{x}(1, t)=U(t), t>0, \\
u(x, 0)=u_{0}(x), u_{t}(x, 0)=u_{1}(x), 0<x<1, \\
y(0)=y_{0}, y^{\prime}(0)=y_{1},
\end{array}\right.
$$

where $u(x, t)$ represents the wave equation's state, $y(t)$ represents the ODE's state. It can describe the vertical displacement of the string and the lower rigid body for the model of two rigid bodies connected by a spring and hanging from an elastic string. $x_{0} \in(0,1)$ is a given intermediate point; $a>0$ and $b \neq 0$ are constants; $U(t)$ is the controller; and $u_{0}(x), u_{1}(x), y_{0}, y_{1}$ are initial data. This system involves two differential equations: a second order ODE in terms of $t$, and a wave PDE in terms of $x$ and $t$. The ODE is subject to initial conditions at $t=0$, and the PDE is subject to boundary conditions at $x=0$ and $x=1$, and initial conditions at $t=0$.

Stabilization of PDEs is a fundamental problem in control theory. Many stabilization approaches have been proposed for PDE control systems, including the control Lyapunov function method, the damping method, the homogeneity method, the return method, the linear quadratic (LQ) method and the backstepping method. These methods can be used to deal with many kinds of PDEs (see [3] and the relevant references therein). The backstepping method, in particular, has several advantages in feedback controller design, such as being easy to understand and implement (see $[4,5,7,9,10,12])$. For the stabilization of PDEs with integral terms, several backstepping methods are available (see $[1,2,6,11,13])$. References $[1,2]$ discuss stabilization via

This work was supported by the Natural Science Foundation of China (grant no. 11301427) and the Fundamental Research Funds for Central Universities in China (grant no. XDJK2014B021). *Author for correspondence. backstepping for ODE-wave and ODE-heat systems with integral terms. Recently, in [13], the stabilization of an ODEheat system in which the heat equation and ODE are coupled at an intermediate point has been considered. Our goal in this paper is to extend the work in [13] to ODE-wave systems in the form of (1).

In system (1), the two differential equations are coupled at an interior point $x_{0} \in(0,1)$ rather than a boundary point. This makes designing the stabilization feedback controller much more difficult than for ODE-wave systems coupled at a boundary point (see [8]).

Generalizations of system (1) in which the term $b u\left(x_{0}, t\right)$ is replaced by a non-local source term, i.e., $\int_{0}^{1} B_{1}(x) u(x, t) d x$, where $B_{1}(\cdot) \in H^{1}(0,1)$, have been discussed in [1]. When $B_{1}(x)=b \delta\left(x-x_{0}\right)$, where $\delta(x)$ is the Dirac function, the non-local source term is exactly $b u\left(x_{0}, t\right)$ as in (1). However, to prove closed-loop stability using the results in [1], it is necessary to validate a complex controllability condition and determine whether certain matrices are invertible. For system (1), these matrices may not be invertible for some choices of $a$ and $b$. Therefore, the results in [1] may not be applicable, motivating the new results to be discussed in this paper. More importantly, our new backstepping transformation yields kernel functions with stronger regularity properties compared with the continuous kernel functions in [1]. The main difference with our stabilization procedure lies in the choice of target system and backstepping transformation. Our choices lead to different conditions for closed-loop stability. The motivation for this paper comes from [1] and the main idea is borrowed from [13]. The novelty of our work lies in determining a suitable feedback matrix in the target system and a corresponding backstepping transformation with smooth kernels, and introducing new techniques to avoid solving coupled kernel equations.

This paper is organized as follows. In Section 2, we show how to design a stabilization feedback vector for system (1) and define a forward backstepping transformation and target system. In Section 3, we prove existence of smooth kernel functions for the forward transformation in Section 2, and design a feedback boundary controller to stabilize the orig- 
inal system. In Section 4, we show that the proposed backstepping transformation is invertible and prove exponential stability of the closed-loop system. In Section 5, we present some simulation results.

\section{Backstepping transformation and target system}

Set $X(t)=\left(y(t), y^{\prime}(t)\right)^{T}$. Then we can rewrite system (1) in matrix form as follows:

$$
\left\{\begin{array}{l}
\dot{X}(t)=A X(t)+B u\left(x_{0}, t\right), t>0, \\
u_{t t}(x, t)=u_{x x}(x, t), 0<x<1, t>0, \\
u(0, t)=0, u_{x}(1, t)=U(t), t>0, \\
u(x, 0)=u_{0}(x), u_{t}(x, 0)=u_{1}(x), 0<x<1, \\
X(0)=X_{0},
\end{array}\right.
$$

where

$$
A=\left(\begin{array}{cc}
0 & 1 \\
a^{2} & 0
\end{array}\right), B=\left(\begin{array}{l}
0 \\
b
\end{array}\right), X_{0}=\left(\begin{array}{l}
y_{0} \\
y_{1}
\end{array}\right) .
$$

Since $\operatorname{rank}(B, A B)=2$, system $[A, B]$ is controllable. In fact, we have the following lemma proved in [13].

Lemma 2.1. For system $[A, B]$, there exists a constant $\mu$ and $a$ vector $K=\mu(a 1)$, which is the left eigenvector corresponding to the positive eigenvalue a of matrix $A$, such that $A+B K$ is exponentially stable.

It is well-known that $[A, B]$ being controllable implies the existence of a vector $K$ such that $A+B K$ is exponentially stable. Lemma 2.1 goes beyond the standard result to give the explicit form of $K$.

To stabilize system (2), we seek a transformation $(X, u) \mapsto(X, w)$ that converts system (2) into an exponentially stable target system. Stability of the closed loop system can then be established in terms of the boundedness of the backstepping transformation and its inverse. We define the backstepping transformation as

$$
\begin{aligned}
& w(x, t)=u(x, t)-\int_{0}^{x} k(x, y) u(y, t) d y \\
& -\int_{0}^{x} l(x, y) u_{t}(y, t) d y-p(x) \int_{0}^{x_{0}} q(y) u(y, t) d y \\
& -p_{1}(x) \int_{0}^{x_{0}} q_{1}(y) u_{t}(y, t) d y-\phi(x) X(t),
\end{aligned}
$$

where the kernels $p(x), q(y), p_{1}(x), q_{1}(y), k(x, y), l(x, y)$ and $\phi(x)$ are to be determined later. The target system is chosen as

$$
\left\{\begin{array}{l}
\dot{X}(t)=(A+B K) X(t)+B w\left(x_{0}, t\right), t>0, \\
w_{t t}(x, t)=w_{x x}(x, t), 0<x<1, t>0, \\
w(0, t)=0, w_{x}(1, t)=-c w_{t}(1, t), t>0, \\
w(x, 0)=w_{0}(x), w_{t}(x, 0)=w_{1}(x), 0<x<1, \\
X(0)=X_{0},
\end{array}\right.
$$

where $c>0$ is a given number, $K$ is as defined in Lemma 2.1 , and $w_{0}(x)$ and $w_{1}(x)$ are the mappings of $u_{0}(x)$ and $u_{1}(x)$ under transformation (3). The well-posedness of (4) is well-known (see [7]). If the kernels in (3) can be chosen to ensure that the transformation $(X, u) \mapsto(X, w)$ maps system (2) into system (4), then the exponential stability of the original system follows from the exponential stability of (4). We now derive the equations for the kernels $p(x), q(y)$, $p_{1}(x), q_{1}(y), k(x, y), l(x, y)$ and $\phi(x)$ in (3).

\section{Kernel equations}

By differentiating $w(x, t)$ in (3) twice with respect to $x$ and $t$, then applying integration by parts, we obtain

$$
\begin{aligned}
& w_{t t}(x, t)-w_{x x}(x, t) \\
&=\left(\phi^{\prime \prime}(x)-\phi(x) A^{2}\right) X(t)-p_{1}(x) q_{1}\left(x_{0}\right) u_{x t}\left(x_{0}, t\right) \\
&+\int_{0}^{x}\left(k_{x x}(x, y)-k_{y y}(x, y)\right) u d y \\
&+\left(l(x, 0)+p_{1}(x) q_{1}(0)\right) u_{x t}(0, t)+2 l^{\prime}(x, x) u_{t}(x, t) \\
&+\int_{0}^{x}\left(l_{x x}(x, y)-l_{y y}(x, y)\right) u_{t} d y-p(x) q\left(x_{0}\right) u_{x}\left(x_{0}, t\right) \\
&+\left(p(x) q^{\prime}\left(x_{0}\right)-\phi(x) A B\right) u\left(x_{0}, t\right) \\
&+\int_{0}^{x_{0}}\left(p^{\prime \prime}(x) q(y)-p(x) q^{\prime \prime}(y)\right) u d y+2 k^{\prime}(x, x) u(x, t) \\
&+\left(p_{1}(x) q_{1}^{\prime}\left(x_{0}\right)-\phi(x) B\right) u_{t}\left(x_{0}, t\right) \\
&+\int_{0}^{x_{0}}\left(p_{1}^{\prime \prime}(x) q_{1}(y)-p_{1}(x) q_{1}^{\prime \prime}(y)\right) u_{t} d y \\
&+(k(x, 0)+p(x) q(0)) u_{x}(0, t),
\end{aligned}
$$

where

$$
k_{x}(x, x)=\left.\frac{\partial k(x, y)}{\partial x}\right|_{y=x}, k_{y}(x, x)=\left.\frac{\partial k(x, y)}{\partial y}\right|_{y=x}
$$

and

$$
k^{\prime}(x, x)=k_{x}(x, x)+k_{y}(x, x) .
$$

According to (3) and $w(0, t)=0$, we know that $u(0, t)-\int_{0}^{x_{0}}\left(p(0) q(y) u+p_{1}(0) q_{1}(y) u_{t}\right) d y-\phi(0) X(t)=0$

We choose

$$
p(0)=0, \quad p_{1}(0)=0, \quad \phi(0)=0 .
$$

Thus, by $w_{x}(1, t)=-c w_{t}(1, t)$ and equations (2) and (3), we obtain the control law

$$
\begin{aligned}
& U(t)=\int_{0}^{1}\left(c l_{y y}(1, y)+k_{x}(1, y)\right) u d y-c l_{y}(1,1) u(1, t) \\
& +\int_{0}^{x_{0}}\left(p_{1}^{\prime}(1) q_{1}(y)+c p(1) q(y)\right) u_{t} d y+\phi^{\prime}(1) X(t) \\
& +\int_{0}^{x_{0}}\left(p^{\prime}(1) q(y)+c p_{1}(1) q_{1}^{\prime \prime}(y)\right) u d y+c \phi(1) A X(t) \\
& +\int_{0}^{1}\left(l_{x}(1, y)+c k(1, y)\right) u_{t} d y-c u_{t}(1, t)
\end{aligned}
$$


which stabilizes the original system. On the other hand, from (2), (3) and (4), we have

$$
\begin{aligned}
& X^{\prime}(t)=A X(t)+B u\left(x_{0}, t\right) \\
& =A X(t)+B\left(w\left(x_{0}, t\right)+\int_{0}^{x_{0}}\left(k\left(x_{0}, y\right) u+l\left(x_{0}, y\right) u_{t}\right) d y\right. \\
& \left.+\int_{0}^{x_{0}}\left(p\left(x_{0}\right) q(y) u+p_{1}\left(x_{0}\right) q_{1}(y) u_{t}\right) d y+\phi\left(x_{0}\right) X(t)\right) \\
& =B \int_{0}^{x_{0}}\left(p\left(x_{0}\right) q(y)+k\left(x_{0}, y\right)\right) u d y+\left(A+B \phi\left(x_{0}\right)\right) X(t) \\
& +B \int_{0}^{x_{0}}\left(l\left(x_{0}, y\right)+p_{1}\left(x_{0}\right) q_{1}(y)\right) u_{t} d y+B w\left(x_{0}, t\right) \\
& =(A+B K) X(t)+B w\left(x_{0}, t\right),
\end{aligned}
$$

if we choose $\phi\left(x_{0}\right)=K, p\left(x_{0}\right) q(y)+k\left(x_{0}, y\right)=0$ and $l\left(x_{0}, y\right)+p_{1}\left(x_{0}\right) q_{1}(y)=0$. Therefore, based on (4), we need to choose $k(x, y), l(x, y), p(x), p_{1}(x), q(y), q_{1}(y)$ and $\phi(x)$ to satisfy

$$
\left\{\begin{array}{l}
k_{x x}(x, y)-k_{y y}(x, y)=0 \\
k(x, x)=0, k(x, 0)=-p(x) q(0) \\
l_{x x}(x, y)-l_{y y}(x, y)=0 \\
l(x, x)=0, l(x, 0)=-p_{1}(x) q_{1}(0) \\
p^{\prime \prime}(x) q(y)-p(x) q^{\prime \prime}(y)=0 \\
q\left(x_{0}\right)=0, p(x) q^{\prime}\left(x_{0}\right)=\phi(x) A B \\
p(0)=0, k\left(x_{0}, y\right)+p\left(x_{0}\right) q(y)=0 \\
p_{1}^{\prime \prime}(x) q_{1}(y)-p_{1}(x) q_{1}^{\prime \prime}(y)=0 \\
q_{1}\left(x_{0}\right)=0, p_{1}(x) q_{1}^{\prime}\left(x_{0}\right)=\phi(x) B \\
p_{1}(0)=0, l\left(x_{0}, y\right)+p_{1}\left(x_{0}\right) q_{1}(y)=0 \\
\phi^{\prime \prime}(x)-\phi(x) A^{2}=0 \\
\phi\left(x_{0}\right)=K, \phi(0)=0
\end{array}\right.
$$

To solve these equations, we first separate them into five subsystems as follows:

$$
\begin{gathered}
\left\{\begin{array}{l}
k_{x x}(x, y)-k_{y y}(x, y)=0, \\
k(x, x)=0, k(x, 0)=-p(x) q(0),
\end{array}\right. \\
\left\{\begin{array}{l}
l_{x x}(x, y)-l_{y y}(x, y)=0, \\
l(x, x)=0, l(x, 0)=-p_{1}(x) q_{1}(0),
\end{array}\right. \\
\left\{\begin{array}{l}
p^{\prime \prime}(x) q(y)-p(x) q^{\prime \prime}(y)=0, \\
q\left(x_{0}\right)=0, p(0)=0,
\end{array}\right. \\
\left\{\begin{array}{l}
p_{1}^{\prime \prime}(x) q_{1}(y)-p_{1}(x) q_{1}^{\prime \prime}(y)=0, \\
q_{1}\left(x_{0}\right)=0, p_{1}(0)=0,
\end{array}\right.
\end{gathered}
$$

and

$$
\left\{\begin{array}{l}
\phi^{\prime \prime}(x)-\phi(x) A^{2}=0 \\
\phi\left(x_{0}\right)=K, \phi(0)=0
\end{array}\right.
$$

with compatibility conditions

$$
\left\{\begin{array}{l}
k\left(x_{0}, y\right)+p\left(x_{0}\right) q(y)=0, \\
l\left(x_{0}, y\right)+p_{1}\left(x_{0}\right) q_{1}(y)=0,
\end{array}\right.
$$

and

$$
\left\{\begin{array}{l}
p(x) q^{\prime}\left(x_{0}\right)=\phi(x) A B, \\
p_{1}(x) q_{1}^{\prime}\left(x_{0}\right)=\phi(x) B .
\end{array}\right.
$$

Next, we solve each subsystem separately to obtain $k(x, y)$, $l(x, y), p(x), p_{1}(x), q(y), q_{1}(y)$ and $\phi(x)$, with the solution parameters chosen to ensure the compatibility conditions (12) and (13) are satisfied.

Using separation of variables, (9) and (10) can be written as

$$
\left\{\begin{aligned}
\frac{p^{\prime \prime}(x)}{p(x)} & =\frac{q^{\prime \prime}(y)}{q(y)}=\gamma=\lambda^{2} \\
q\left(x_{0}\right) & =0, p(0)=0,
\end{aligned}\right.
$$

and

$$
\left\{\begin{array}{l}
\frac{p_{1}^{\prime \prime}(x)}{p_{1}(x)}=\frac{q_{1}^{\prime \prime}(y)}{q_{1}(y)}=\gamma_{1}=\lambda_{1}^{2}, \\
q_{1}\left(x_{0}\right)=0, p_{1}(0)=0,
\end{array}\right.
$$

According to (11) and (13), we know parameters $\gamma=\lambda^{2}>$ 0 and $\gamma_{1}=\lambda_{1}^{2}>0$. Equations (14) and (15) can be solved explicitly as

$$
\left\{\begin{array}{l}
p(x)=\xi e^{\lambda x}-\xi e^{-\lambda x} \\
q(y)=\eta e^{\lambda y}-\eta e^{\lambda\left(2 x_{0}-y\right)},
\end{array}\right.
$$

and

$$
\left\{\begin{array}{l}
p_{1}(x)=\xi_{1} e^{\lambda_{1} x}-\xi_{1} e^{-\lambda_{1} x} \\
q_{1}(y)=\eta_{1} e^{\lambda_{1} y}-\eta_{1} e^{\lambda_{1}\left(2 x_{0}-y\right)}
\end{array}\right.
$$

where $\xi, \eta, \lambda, \xi_{1}, \eta_{1}$ and $\lambda_{1}$ are parameters to be determined later. Thus, the solutions of (7) and (8) are

$$
\begin{aligned}
& k(x, y)=-\xi \eta\left(1-e^{2 \lambda x_{0}}\right)\left(e^{\lambda(x-y)}-e^{-\lambda(x-y)}\right), \\
& l(x, y)=-\xi_{1} \eta_{1}\left(1-e^{2 \lambda_{1} x_{0}}\right)\left(e^{\lambda_{1}(x-y)}-e^{\lambda_{1}(y-x)}\right) .
\end{aligned}
$$

We now need to choose suitable values for the parameters $\xi, \eta, \lambda, \xi_{1}, \eta_{1}$ and $\lambda_{1}$ so that the compatibility conditions (12) and (13) are satisfied. From the first equation in (12),

$$
\begin{aligned}
& p\left(x_{0}\right) q(y)+k\left(x_{0}, y\right) \\
& =\xi \eta\left(e^{\lambda x_{0}}-e^{-\lambda x_{0}}\right)\left(e^{\lambda y}-e^{\lambda\left(2 x_{0}-y\right)}\right) \\
& \quad-\xi \eta\left(1-e^{2 \lambda x_{0}}\right)\left(e^{\lambda\left(x_{0}-y\right)}-e^{-\lambda\left(x_{0}-y\right)}\right) \\
& =0 .
\end{aligned}
$$

Similarly, from the second equation in (12),

$$
p_{1}\left(x_{0}\right) q_{1}(y)+l\left(x_{0}, y\right)=0 \text {. }
$$

From the first equations in (13) and (16) and $\phi\left(x_{0}\right)=K$, we have

$$
\begin{aligned}
& p\left(x_{0}\right) q^{\prime}\left(x_{0}\right)-\phi\left(x_{0}\right) A B \\
& =2 \lambda \xi \eta e^{\lambda x_{0}}\left(e^{\lambda x_{0}}-e^{-\lambda x_{0}}\right)-K A B=0,
\end{aligned}
$$

which holds when

$$
\xi \eta=\frac{K A B}{2 \lambda e^{\lambda x_{0}}\left(e^{\lambda x_{0}}-e^{-\lambda x_{0}}\right)} .
$$

Similarly, using the second equation in (13) and (17), we choose

$$
\xi_{1} \eta_{1}=\frac{K B}{2 \lambda_{1} e^{\lambda_{1} x_{0}}\left(e^{\lambda_{1} x_{0}}-e^{-\lambda_{1} x_{0}}\right)} .
$$

When $\xi \eta$ and $\xi_{1} \eta_{1}$ are determined by (18) and (19), $k(x, y), l(x, y), p(x) q(y)$ and $p_{1}(x) q_{1}(y)$ are determined uniquely. Meanwhile, the compatibility condition (13) at 
$x=x_{0}$ is satisfied. Substituting (18) and (19) into (13), we have

$$
\phi(x)=\frac{e^{\lambda x}-e^{-\lambda x}}{e^{\lambda x_{0}}-e^{-\lambda x_{0}}} K=\frac{e^{\lambda_{1} x}-e^{-\lambda_{1} x}}{e^{\lambda_{1} x_{0}}-e^{-\lambda_{1} x_{0}}} K .
$$

Therefore, if $\lambda=\lambda_{1}$, compatibility conditions (13) hold. By differentiating (20) twice with respect to $x$, we obtain

$$
\phi^{\prime \prime}(x)=\lambda^{2} \frac{e^{\lambda x}-e^{-\lambda x}}{e^{\lambda x_{0}}+e^{-\lambda x_{0}}} K
$$

Substituting $\phi(x)$ and $\phi^{\prime \prime}(x)$ into (11), we see that $\lambda$ needs to satisfy $\lambda^{2} K-K A^{2}=0$, which holds when $\lambda^{2}=a^{2}$. Hence, by setting $\mathbb{T}:=\left\{(x, y) \in \mathbb{R}^{2} \mid 0 \leq x \leq 1,0 \leq y \leq x\right\}$, we have the following existence theorem for smooth kernel functions.

Theorem 3.1. Equations (6) have unique smooth solution$s k(\cdot, \cdot) \in C^{2}(\mathbb{T}), l(\cdot, \cdot) \in C^{2}(\mathbb{T}), p(\cdot) q(\cdot) \in C^{2}([0,1] \times$ $\left.\left[0, x_{0}\right]\right), p_{1}(\cdot) q_{1}(\cdot) \in C^{2}\left([0,1] \times\left[0, x_{0}\right]\right)$ and $\phi(\cdot) \in$ $C^{2}([0,1])$.

\section{Inverse transformation and closed-loop stability}

We now show that the backstepping transformation (3) is invertible. The inverse transformation of (3) can be written as

$$
\begin{aligned}
& u(x, t) \\
& =w(x, t)+\int_{0}^{x} n(x, y) w(y, t) d y+\int_{0}^{x} m(x, y) w_{t}(y, t) d y \\
& +g(x) \int_{0}^{x_{0}} h(y) w(y, t) d y+g_{1}(x) \int_{0}^{x_{0}} h_{1}(y) w_{t}(y, t) d y \\
& +\psi(x) X(t)
\end{aligned}
$$

where functions $m(x, y), n(x, y), g(x), h(y), g_{1}(x), h_{1}(y)$ and $\psi(x)$ are to be determined later. Following the same procedure as in Section 4 and applying $u(0, t)=0$ and equations (4) and (21), we obtain

$$
\int_{0}^{x_{0}}\left(g(0) h(y) w+g_{1}(0) h_{1}(y) w_{t}\right) d y+\psi(0) X(t)=0 .
$$

Thus,

$$
g(0)=0, g_{1}(0)=0, \psi(0)=0
$$

Finally, we have

$$
\begin{aligned}
& X^{\prime}(t)=A X(t)+B u\left(x_{0}, t\right) \\
& =A X+B\left(w\left(x_{0}, t\right)+\int_{0}^{x_{0}}\left(n\left(x_{0}, y\right) w+m\left(x_{0}, y\right) w_{t}\right) d y\right. \\
& \left.+\int_{0}^{x_{0}}\left(g\left(x_{0}\right) h(y) w+g_{1}\left(x_{0}\right) h_{1}(y) w_{t}\right) d y+\psi\left(x_{0}\right) X(t)\right) \\
& =(A+B K) X(t)+B w\left(x_{0}, t\right),
\end{aligned}
$$

after choosing $\psi\left(x_{0}\right)=K, n\left(x_{0}, y\right)+g\left(x_{0}\right) h(y)=0$ and $m\left(x_{0}, y\right)+g_{1}\left(x_{0}\right) h_{1}(y)=0$. To summarize, we need to choose the kernels $m(x, y), n(x, y), g(x), h(y), g_{1}(x)$, $h_{1}(y)$ and $\psi(x)$ to satisfy

$$
\left\{\begin{array}{l}
n_{x x}(x, y)-n_{y y}(x, y)=0, \\
n(x, x)=0, n(x, 0)=-g(x) h(0), \\
m_{x x}(x, y)-m_{y y}(x, y)=0, \\
m(x, x)=0, m(x, 0)=-g_{1}(x) h_{1}(0), \\
g^{\prime \prime}(x) h(y)-g(x) h^{\prime \prime}(y)=0, \\
h\left(x_{0}\right)=0, g(x) h^{\prime}\left(x_{0}\right)=\psi(x)(A+B K) B, \\
g(0)=0, g\left(x_{0}\right) h(y)+n\left(x_{0}, y\right)=0, \\
g_{1}^{\prime \prime}(x) h_{1}(y)-g_{1}(x) h_{1}^{\prime \prime}(y)=0, \\
h_{1}\left(x_{0}\right)=0, g_{1}(x) h_{1}^{\prime}\left(x_{0}\right)=\psi(x) B, \\
g_{1}(0)=0, g_{1}\left(x_{0}\right) h_{1}(y)+m\left(x_{0}, y\right)=0, \\
\psi^{\prime \prime}(x)-\psi(x)(A+B K)^{2}=0, \\
\psi\left(x_{0}\right)=K, \psi(0)=0 .
\end{array}\right.
$$

Using the same solution as for (6), we obtain the following existence theorem.

Theorem 4.1. Equations (22) have unique smooth solutions $m(\cdot, \cdot) \in C^{2}(\mathbb{T}), n(\cdot, \cdot) \in C^{2}(\mathbb{T}), g(\cdot) h(\cdot) \in$ $C^{2}\left([0,1] \times\left[0, x_{0}\right]\right), g_{1}(\cdot) h_{1}(\cdot) \in C^{2}\left([0,1] \times\left[0, x_{0}\right]\right)$ and $\psi(\cdot) \in C^{2}([0,1])$.

Theorem 4.2. For the closed-loop system (2) with control law (5), if the initial conditions $u_{x}(\cdot, 0)$ and $u_{t}(\cdot, 0)$ are square integrable in $x$ and compatible with (3), then the closed-loop system has a unique solution in $\left(u(\cdot, \cdot), u_{t}(\cdot, \cdot), X(\cdot)\right) \in C\left([0,+\infty) ; H^{1}(0,1) \times L^{2}(0,1)\right) \times$ $C^{1}[0,+\infty)$, which is exponentially stable in the sense that there exists constants $\sigma>0$ and $\omega>0$ such that

$$
\begin{aligned}
& \|X(t)\|^{2}+\left\|u_{t}(\cdot, t)\right\|_{2}^{2}+\left\|u_{x}(\cdot, t)\right\|_{2}^{2} \\
& \leq \sigma\left(\|X(0)\|^{2}+\left\|u_{t}(\cdot, 0)\right\|_{2}^{2}+\left\|u_{x}(\cdot, 0)\right\|_{2}^{2}\right) e^{-\omega t},
\end{aligned}
$$

where

$$
\|u(\cdot, t)\|_{2}:=\left(\int_{0}^{1} u(x, t)^{2} d x\right)^{\frac{1}{2}}
$$

and $\|\cdot\|$ denotes the Euclidean norm.

Proof. This result follows from the exponential stability of target system (4) and the inverse transformation (21).

\section{Numerical simulations}

We simulated the closed-loop system for the following data: $x_{0}=0.5, \mu=-2, a=1, b=1, c=1, y_{0}=1, y_{1}=0$, $w_{0}(x)=10(1-x)^{2}, w_{1}(x)=(1-x) x$. The initial functions $u_{0}(x)$ and $u_{1}(x)$ were obtained by applying the inverse transformation $(21)$ to $w_{0}(x)$ and $w_{1}(x)$. The simulation results for system (1) with $U(t)=0$ and feedback control law (5) are presented respectively in Figures 1-5. Figure 1 shows the solution of the ODE with $U(t)=0$, and Figure 2 shows the solution of the wave equation with $U(t)=0$. Figure 3 shows the stabilizing controller $U(t)$, Figure 4, 5 show the solution of the ODE and wave equation respectively with feedback control law (5). Figures 4 and 5 clearly show that the closed-loop system is stable as $t \longrightarrow+\infty$, thus verifying the theoretical results obtained in the previous sections. 


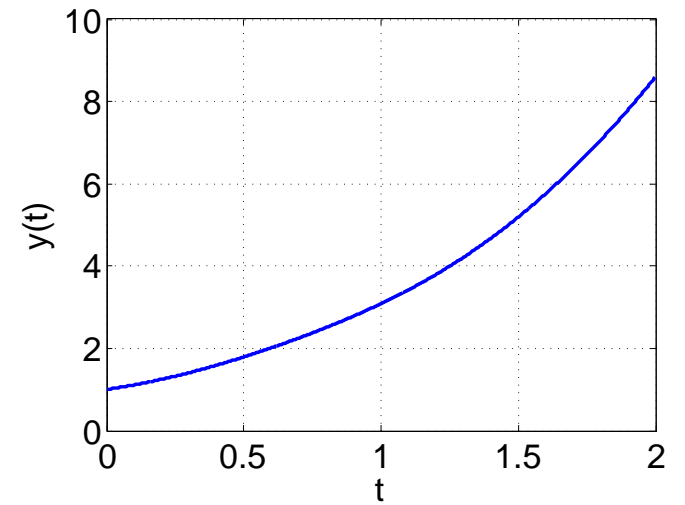

Fig. 1: State of the ODE without controller.

Fig. 2: State of the wave equation without controller.

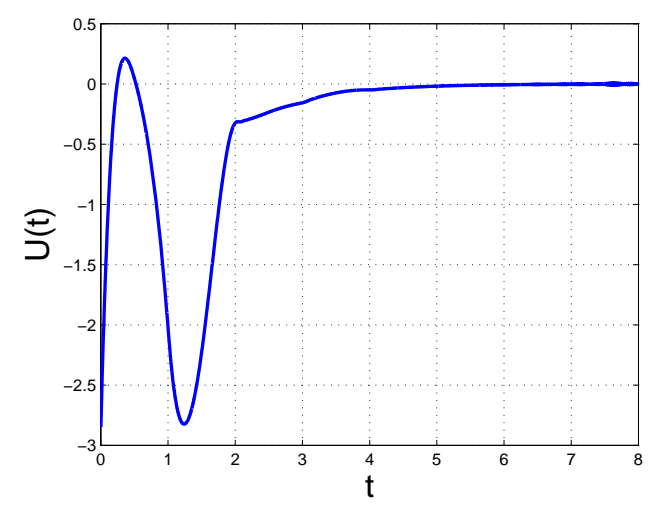

Fig. 3: Stabilizing controller $U(t)$.

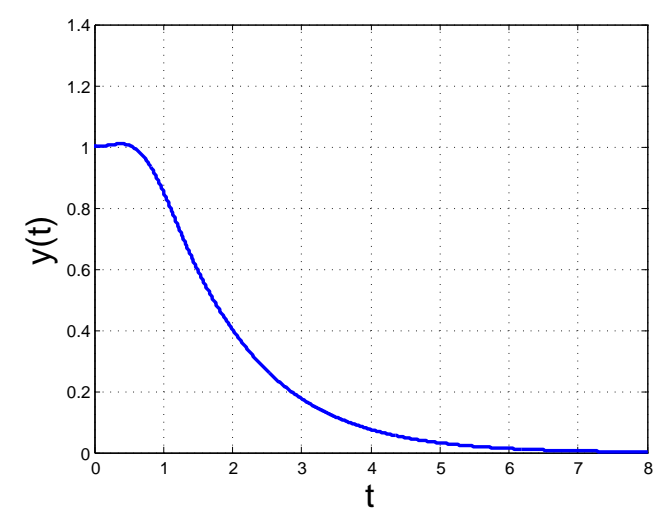

Fig. 4: Closed-loop response of the ODE.

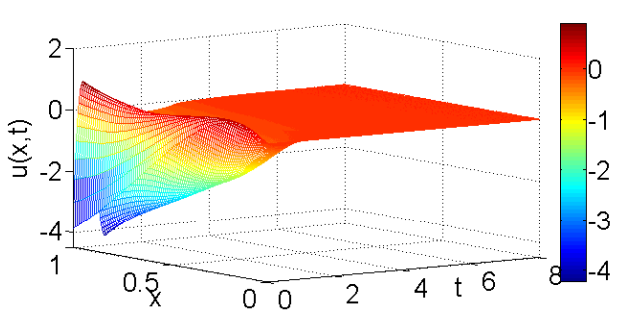

Fig. 5: Closed-loop response of the wave equation.

\section{References}

[1] N. Bekiaris-Liberis and M. Krstic, Compensating the distributed effect of a wave PDE in the actuation or sensing path of MIMO LTI systems. Systems and Control Letters, 59, 713 719, 2010.

[2] N. Bekiaris-Liberis and M. Krstic, Compensating the distributed effect of diffusion and counter-convection in multiinput and multi-output LTI systems. IEEE Transactions on Automatic Control, 56, 637-643, 2011.

[3] J. M. Coron, Control and nonlinearity. American Mathematical Society, 2007.

[4] J. M. Coron and Q. Lü, Local rapid stabilization for a Korteweg-de Vries equation with a Neumann boundary control on the right, Journal de Mathéatiques Pures et Appliquées, 102, 1080-1120, 2014.

[5] J. M. Coron, R. Vazquez, M. Krstic and G. Bastin, Local exponential $H^{2}$ stabilization of a $2 \times 2$ quasilinear hyperbolic system using backstepping, SIAM Journal of Control and Optimization, 51, 2005-2035, 2013.

[6] C. Guo, C. Xie and Z. Zhou, Stabilization of a spatially noncausal reaction-diffusion equation by boundary control, International Journal of Robust and Nonlinear Control, 24, 117, 2014.

[7] M. Krstic, B. Guo, A. Blogh and A. Smyshlyaev, Output feedback stabilization of an unstable wave equation, Automatica, 
44, 63-74, 2008.

[8] M. Krstic and A. Smyshlyaev, Boundary control of PDEs: A course on backstepping designs, SIAM, 2008.

[9] A. Smyshlyaev, E. Cerpa and M. Krstic, Boundary stabilization of a 1-D wave equation with in-domain anti-damping, SIAM Journal of Control and Optimization, 48, 4014-4031, 2010.

[10] G. Susto and M. Krstic, Control of PDE-ODE cascades with Neumann interconnections, Journal of the Franklin Institute, 347, 284-314, 2010.

[11] Z. Zhou and C. Guo, Stabilization of linear heat equation with a heat source at intermediate point by boundary control, $\mathrm{Au}$ tomatica, 49, 448-456, 2013.

[12] Z. Zhou and S. Tang, Boundary stabilization of a coupled wave-ODE system with internal anti-damping, International Journal of Control, 85, 1683-1693, 2012.

[13] Z. Zhou and C. Xu, Stabilization of a second order ODE-heat system coupling at intermediate point, Automatica, 60, 5764, 2015. 